\title{
THE CONJUGACY PROBLEM AND CYCLIC AMALGAMATIONS
}

\author{
BY SEYMOUR LIPSCHUTZ
}

Communicated by Dock S. Rim, July 14, 1974

Max Dehn first posed the word and conjugacy problems for groups, and solved these problems [2] for the fundamental group $G_{k}$ for an orientable 2-manifold of genus $k$. This group has the presentation

$$
G_{k}=\left(a_{1}, b_{1}, \cdots, a_{k}, b_{k} ; a_{1}^{-1} b_{1}^{-1} a_{1} b_{1} \cdots a_{k}^{-1} b_{k}^{-1} a_{k} b_{k}=1\right) .
$$

We note that $G_{k}$ is a free product of two free groups with a cyclic amalgamation generated by nonpowers.

The author generalized Dehn's result [3] by solving the conjugacy problem for any free product of free groups with a cyclic amalgamation. On the other hand, Miller [5] gave an example of a free product of two free groups amalgamating finitely generated subgroups which has an unsolvable conjugacy problem. Thus a cyclic amalgamation seems an essential criteria in finding classes of groups with solvable conjugacy problems. (For notational convenience we will speak of a free product "amalgamating $u$ and $v$ " when we mean "amalgamating the cyclic subgroups generated by $u$ and $v$ ".)

Anshel and Stebe solved the conjugacy problem [1] for certain $H N N$ extensions where the underlying group is free and the extension is obtained by an isomorphism of cyclic subgroups. Following Anshel and Stebe, we say that an element $h$ in a group $G$ is non-self-conjugate if its distinct powers are in different conjugacy classes. We will also say that $h$ is power-solvable if for any $w$ in $G$ we can decide whether or not $w$ is a power of $h$. (A group has a solvable power problem if all its elements are power-solvable.) We note that every nonidentity element in a free group is non-self-conjugate and powersolvable.

We now are able to state our main result which clearly generalizes Dehn's result.

AMS (MOS) subject classifications (1970). Primary 20F05; Secondary 20E05.

Key words and phrases. Conjugacy problem, free product with amalgamation.

Copyright $\odot$ 1975, American Mathematical Society 
THEOREM 1. Let $u$ be any nonpower in a free group $A$. Let $v$ be a non-self-conjugate and power-solvable element in a group $B$ with a solvable conjugacy problem. Then the free product of $A$ and $B$ amalgamating $u$ and $v$ has a solvable conjugacy problem.

The requirement that $u$ be a nonpower in a free group $A$ is actually stronger than needed. In order to concisely restate Theorem 1 in a more general form, we introduce a definition. An element $h$ in a group $G$ will be called a critical element if it has the following four properties:

(a) $h$ is non-self-conjugate.

(b) $h$ is conjugate-power-solvable, i.e. for any $w$ in $G$ we can decide whether or not $w$ is conjugate to a power of $h$.

(c) $h$ is double-coset-solvable, i.e. for any pair $u, v$ in $G$ we can decide whether or not there exist integers $r$ and $s$ such that $h^{r} u h^{s}=v$.

(d) If $h^{m} u=u h^{m}$, then $u$ is a power of $h$.

We will also say that $h$ is semicritical if $h$ satisfies the first three of the four properties.

The author's main technical result in [3] shows that elements in free groups are double-coset-solvable. Such elements are clearly conjugate-powersolvable. Nonpowers also satisfy property (d). Hence nonidentity elements in a free group are semicritical, and nonpowers are critical. Accordingly, the following two theorems generalize Theorem 1 and the main result in [3].

THEOREM 2. Let $A$ and $B$ be groups with solvable conjugacy problems. Let $u$ be a critical element of $A$ and let $v$ be a non-self-conjugate and power-solvable element of $B$. Then the free product of $A$ and $B$ amalgamating $u$ and $v$ has a solvable conjugacy problem.

THEOREM 3. Let $G$ be the free product of groups with solvable conjugacy problem amalgamating a cyclic subgroup generated by semicritical elements in the factors. Then $G$ has a solvable conjugacy problem.

All relevent terms appear in the text by Magnus, Karrass and Solitar [4]. Details and proofs of the above results will appear elsewhere.

\section{REFERENCES}

1. M. Anshel and P. Stebe, The solvability of the conjugacy problem for certain HNN groups, Bull. Amer. Math. Soc. 80 (1974), 266-269.

2. M. Dehn, Transformation der Kurven auf Zweiseitigen Flöchen, Math. Ann. 72 (1912), 413-421. 
3. S. Lipschutz, Generalization of Dehn's result on the conjugacy problem, Proc. Amer. Math. Soc. 17 (1966), 759-762. MR 33 \#5706.

4. W. Magnus, A. Karrass and D. Solitar, Combinatorial group theory: Presentations of groups in terms of generators and relations, Pure and Appl. Math., vol. 13, Interscience, New York, 1966. MR 34 \#7617.

5. C. F. Miller III, On group-theoretic decision problems and their classifications, Ann. of Math. Studies, no. 68, Princeton Univ. Press, Princeton, N. J.; Univ. of Tokyo Press, Tokyo, 1971. MR 46 \#9147.

DEPARTMENT OF MATHEMATICS, TEMPLE UNIVERSITY, PHILADELPHIA, PENNSYLVANIA 19122 